\title{
Die andere Seite Kakaniens Österreichische Phantastik nach 1900
}

\section{Clemens Ruthner}

Ein Wahlplakat aus den frühen zwanziger Jahren, 1995 in Brüssel ausgestellt, zeigt eine geisterhafte Armee in Weltkriegsmontur, Knochenschädel unter den Stahlhelmen. Der dazugehörige Slogan lautet: "Die Toten rufen! Sind wir umsonst gestorben? Wählt die Sozialdemokraten, die zur Freiheit führen!"

Das Sujet verlangt eine Rechtfertigung der Kriegsopfer, gleichzeitig den apotropäischen Schutz vor ihnen. Auslöser einer angepeilten politischen Befreiung wäre demnach die dräuende Freisetzung der Toten - dies alles veranstaltet von einer künstlerischen Freiheit, die sich des Todes bemächtigt hat. Ein Beispiel unter vielen, das veranschaulicht, welch suggestive Verbindung das Unheimlich-Irrationale und eine politische Rhetorik im Zeitgeist des frühen zwanzigsten Jahrhunderts eingehen können. Aber ist jene auffallende Fluktuation jenseitiger Bilder in der ideologischen Phantasie des Diesseits um 1918 einzig hervorgerufen durch den Weltkrieg?

Der hier zugrundeliegende phantastische Diskurs behauptet - folgen wir den Theorien von Freud (1919) ${ }^{1}$ und Todorov $^{2}$ - das Hineinwirken eines ausgetriebenen Übernatürlichen in unsere aufgeklärt materialistische Welt. Das so entstandene historische Genre hatte in der Literatur ${ }^{3}$ seine erste literarische Hochkonjunktur im Gefolge der Aufklärung bis hin zu den Ausklängen der Romantik und Post-Romantik; hierin begriffen sind im deutschsprachigen Raum auch die Blüte des Wiener Volks- und Zau-

${ }^{1}$ Sigmund Freud: "Das Unheimliche" in: ders.: Studienausgabe, hrsg. von A. Mitscherlich u.a., Frankfurt a.M. 1985, Bd. IV, S. 243-274.

${ }^{2}$ Tzvetan Todorov: Introduction à la littérature fantastique, Paris 1970.

${ }^{3}$ cf. Rein A. Zondergeld: Lexikon der phantastischen Literatur, Frankfurt a.M. 1983; Joachim Körber ( $\mathrm{Hg}$.): Bibliographisches Lexikon der utopisch-phantastischen Literatur, Meitingen 1984ff. [Loseblattsammlung]; Antoine Faivre: "Genèse d'un genre narratif, le fantastique (essai de périodisation) in: La littérature fantastique. Colloque de Cerisy. Hg. von Antoine Faivre, Paris 1991 [Cahiers de l'Hermétisme]; Neil Corn-well: The Literary Fantastic. From Gothic to Postmodernism, New York u.a. 1990. 
berstücks sowie beispielsweise Grillparzers Ahnfrau-Drama von 1817. Um 1850 wird das Genre unzeitgemäß, klingt epigonal aus bzw. wird im vorherrschenden Positivismus in den Untergrund abgedrängt, ${ }^{4}$ um erst wieder zur Jahrhundertwende sein Comeback im literarischen Mainstream zu feiern, insbesondere auf den Territorien der Monarchie Österreich-Ungarn. ${ }^{5}$

Nach einer zweiten Blütezeit zwischen 1910 und 1930 setzt in Deutschland und Österreich schließlich der Nationalsozialismus der Literatur des Imaginären neuerlich ein Ende. Von den braunen Machthabern wird sie maximal in Form literarischer Folklore geduldet, generell aber eher dem "entarteten" Schrifttum zugeschlagen. ${ }^{6}$ Erst nach 1945 kommt es zu einer begrenzten Wiederaufnahme phantastischen Erzählens in Österreich, wenngleich meist unter anderen - modernistischen bis postmodernistischen - Vorzeichen, etwa beim Österreicher H. C. Artmann (Dracula Dracula, 1966); Zeugnis hierfür geben die Anthologien Der gewöhnliche Schrecken (1969) von Peter Handke und L'Autriche fantastique (1976) von Jean Gyory. ${ }^{7}$ Eine Substratkultur des Irrationalen, wie sie

${ }^{4}$ Dieser erforschenswerte Konjunktureinbruch im neunzehnten Jahrhundert ist ein Charakteristikum des deutschsprachigen Raums gegenüber einer kontinuierlichen Produktion in anderen Ländern: In Frankreich etwa mit Balzac, Gautier, Merimeé, Maupassant, Nerval, Nodier und Villiers de l'Isle Adam, in England mit einer Traditionslinie, die von Lord Byron über Sheridan Le Fanu in die viktorianische Phantastik eines Bram Stoker reicht; nicht zu vergessen die russische Literatur - Gogol, Tolstoi, Turgenjew, Dostojewski - und den Einfluß des Amerikaners E. A. Poe.

5 cf. die Überblicke von Winfried Freund: "Von der Aggression zur Angst. Zur Entwicklung der phantastischen Novellistik in Deutschland" in: Phaicon 3 (1978), S. 9-31 und Jens Malte Fischer "Deutschsprachige Phantastik zwischen Décadence und Faschismus" in: Phaicon 3 (1978), S. 93-130.

${ }^{6}$ Die Verdammung der Phantastik fußt freilich auf keinem exklusiv faschitischen Vorurteil; in den über zweihundert Jahren seiner Geschichte sieht sich das Genre immer wieder von Kritikerpäpsten aller couleurs als "Schundliteratur" verfolgt.

${ }^{7}$ cf. Heinz Harro Kühnelt: "Die Entfaltung der österreichischen grotesken und phantastischen Literatur im 20. Jahrhundert" in: Herbert Zeman (Hg.): Die österreichische Literatur. Eine Dokumentation zu ihrer literarhistorischen Entwicklung, Graz 1986ff., Bd. IV/1, S. 833-846 und Clemens Ruthner: "Wort-Magie. Glossen zum 'phantastischen' Erzählen nach 1945 (H. C. Artmann, Th. Bernhard)" in: Karlheinz Auckenthaler (Hg.): Die Zeit und die Schrift. Österreichische Literatur nach 1945, 
in der Zeit zwischen Romantik und Biedermeier oder zur Jahrhundertwende bestand, scheint aber mit dem Dritten Reich untergegangen.

Es bedurfte offensichtlich des Gesellschaftsklimas der siebziger und achtziger Jahre, um eine neuerliche Renaissance jenes Imaginären manifest zu machen. Unter dem Eindruck zeitgenössischer lateinamerikanischer Literatur (v.a. Jorge Luis Borges, Gabriel García Márquez und Carlos Fuentes) sowie poststrukturalistischer französischer Philosophie sind wir anscheinend nolens volens von neuem in ein phantastisches Zeitalter eingetreten - warum, bleibt noch zu klären. In den Buchläden stapeln sich erfolgreiche Qualitätsprodukte wie Christoph Ransmayrs Letzte Welt (1988) oder Elfriede Jelineks Vampirdrama Krankheit oder Moderne Frauen (1984), während sich im Kino wieder Frankenstein und Dracula Gute Nacht sagen und das Fernsehen in den X-Files blättert. Die dunklen Spiegel, die Phantastik in der Nachfolge der Romantik vorhält, sind so in unserer postmodernen Wißbegier längst verdoppelt, ja zum Spiegelkabinett geworden, in dem sich drei Jahrhundertwenden und ihre Phantasmen begegnen und reflektieren - um es einmal ganz im Sinne von Jorge Luis Borges (1899-1986), dem argentinischen Großmeister des Imaginären, metaphorisch anzudenken.

Eine unter diesen Umständen anlaufende philologische Auseinandersetzung mit dem früher marginalisierten Genre der phantastischen Literatur stellt aber auch eine einmalige Chance dar, ein besseres Verständnis der österreichischen Kultur im frühen zwanzigsten Jahrhundert zu wecken, indem sie deren klärungsbedürftige Abgründe aufzeigt. Denn jene deutschsprachige Belletristik zwischen 1900 und 1930 ist nicht nur erfüllt von modernistischen Experimenten und bürgerlicher Pathographie, süßen Mädeln, fatalen und fragilen Frauen, dekadenten Helden, Männern ohne Eigenschaften und Überich-Beschädigten, in ihr tummeln sich auch Revenants, ${ }^{8}$ Golems, Vampire, Dämonen, ambivalente Fremde und hypnotische Machtmenschen, Larven und Lemuren (K. Kraus!), kurz gesagt: das Bestiarium des Volksglaubens, die Phantasmagorien der Weltreligionen, der literarischen Tradition ${ }^{9}$ und einer modischen Esoterik, ${ }^{10}$ welche die

Szeged 1993, S. 175-185.

8 cf. Clemens Ruthner: Unheimliche Wiederkehr. Interpretationen zu den gespenstischen Romanfiguren bei Ewers, Meyrink, Soyka, Spunda und Strobl, Meitingen 1993.

${ }^{9}$ cf. Ingeborg Vetter: Das Erbe der "Schwarzen Romantik" in der deutschen 
Jahrhundertwende und Zwischenkriegszeit ebenso prägen wie die Unternehmungen der Wiener, Berliner und Münchner Moderne.

In seinem Standardwerk über das Groteske schreibt Wolfgang Kayser, daß man nach dem Zweiten Weltkrieg die Dichter aus dem unheimlichen Prager Umfeld weitgehend vergessen habe, so "daß Kafka heute [...] als der in seiner Zeit Einzigartige gilt". Um 1920 hätte man "seine gewiß eigenartige Stimme noch im Chor der Gleichzeitigen"11 wahrgenommen - ein Befund, dem man zustimmen sollte?

So sorgt denn auch die (Nicht-)Zugehörigkeit der Hochliteratur-Ikone Kafka zur als "trivial" verschrieenen Phantastik nach $1900^{12}$ noch immer für erregte Gemüter: Warum etwa polemisiert Adorno in einem Brief vom 21. Februar 1964 an Peter Szondi so heftig gegen "jene Sphäre literarhistorischen Denkens, in der man dann Kubin mit Kafka zusammenbringt"?

Das in der Generation vor Kafka entstandene Novellengenre der sog. Seltsamen Geschichte zeigt sich freilich eklektisch offen für Figureninventare und Ereignisse aller Art, ${ }^{13}$ worauf auch die unsichere Termino-

Décadence. Studien zur Horrorgeschichte um 1900, Graz 1976 [Diss. masch.]; Peter Cersowsky: Phantastische Literatur im ersten Viertel des 20. Jahrhunderts. Untersuchungen zum Strukturwandel des Genres, seinen geistesgeschichtlichen Voraussetzungen und zur Tradition der "schwarzen Romantik" insbesondere bei Gustav Meyrink, Alfred Kubin und Franz Kafka, München 1983; Hans Richard Brittnacher: Ästhetik des Horrors. Gespenster, Vampire, Monster, Teufel und künstliche Menschen in der phantastischen Literatur, Frankfurt a. M. 1994.

${ }^{10}$ Marianne Wünsch: Die Fantastische Literatur der Frühen Moderne (18901930). Definitionen. Denkgeschichtlicher Kontext. Strukturen, München 1991.

${ }^{11}$ Wolfgang Kayser: Das Groteske. Seine Gestaltung in Malerei und Dichtung, Oldenburg, Hamburg 1957, S. 157.

12 cf. Peter Cersowsky: a.a.O., S. $27 \mathrm{ff}$.

In Franz Kafkas Erzählung Die Verwandlung (1915) etwa löst das Faktum, daß der Held Gregor Samsa sich in einen Käfer verwandelt hat, keinerlei "hésitation" im Sinne Todorovs aus, wieweit die hier vorgestellten Phänomene mit einer naturwissenschaftlichen Weltsicht vereinbar sind. Eine groteske Parallelität und der domestizierte Alltag haben sich in mysteriöser Manier gegenseitig durchdrungen; die Verstörung beim Leser bewirkt nicht die Verwandlung selbst, sondern eher die Reaktion von Samsa und seiner Umwelt.

${ }^{13}$ cf. Ingeborg Vetter: a.a.O. 
logie der Autoren und Philologen hinweist, die zwischen "bizarr", "grotesk", "sonderbar", "phantastisch" u.a. Adjektiven schwanken. Diese Unfähigkeit "to hit upon one definitive designation"14 sollte in bezug auf die Geschlossenheit oder Vagheit des Phantastik-Terms und verwandter Begriffs einmal genauer befragt werden. Man denke etwa an Gustav Meyrinks Beiträge für die Münchner Zeitschrift Simplicissimus: eine eigentümliche Melange aus Phantastik, Groteske, Satire und Travestie, die sich nicht festlegen läßt und sich eher des Instrumentariums von Bachtin als jenem Todorovs würdig erweisen würde. ${ }^{15}$

Auffällig ist jedenfalls der große Anteil an Autoren aus der Donaumonarchie bei der Produktion deutschsprachiger Phantastik im frühen zwanzigsten Jahrhundert. Dies mag mit der häufig problematisierten Kontinuität von barockem Gedankengut zu tun haben, die sich im achtzehnten und neunzehnten Jahrhundert nicht so wie in Deutschland durch den Wirklichkeitssinn von protestantischer Aufklärung, Klassik bzw. Naturalismus gebremst sieht. Es wäre also durchaus denkbar, einen Zusammenhang - wenn schon nicht mit gegenreformatorischen, so doch mit bodenständigen Traditionen des Wunderbaren, etwa dem volkstümlichen Wiener Zauberstück, anzunehmen. ${ }^{16}$

Um die Jahrhundertwende beginnt, wie angedeutet, ein großangelegter Boom von Anthologien jener Seltsamen Geschichten, so z.B. jene des gebürtigen Lembergers Bodo Wildberg (1862-1942), die von 1894 an publiziert werden, ${ }^{17}$ oder eben die legendäre Sammlung Der heiße Soldat u. a. Geschichten (1903) von Gustav Meyrink (1868-1932). Der gebürtige Iglauer Karl Hans Strobl (1877-1946) indes nimmt für sich mit seinem "programmatischen Skizzenbuch" Aus Gründen und Abgrïnden (1901) "in Anspruch, der Erneuerer der phantastischen Erzählung in

${ }^{14}$ Verna Schuetz: The Bizarre Literature of H. H. Ewers, A. Kubin, G. Meyrink and K. H. Strobl, Madison 1974 [Diss. masch.], S. 9.

15 cf. Siegfried Schödel: Studien zu den phantastischen Erzählungen Gustav Meyrinks, Erlangen 1965 [Diss. masch.]; Helga Abret: Gustav Meyrink conteur, Bern u.a. 1976; Jean Jacques Pollet: Essai sur la littérature du début du XXe siècle (1900-1930), Lille 1986 [Diss. Microfiche], S. 264ff.

${ }^{16}$ vgl. Harro Heinz Kühnelt: "E. A. Poe und die phantastische Erzählung im österreichischen Schrifttum von 1900-1920" in: Festschrift für Moriz Enzinger. Hg. von Herbert Seidler, Innsbruck 1953, S. $132 \mathrm{f}$.

${ }^{17}$ Robert N. Bloch: Nachwort zu: Jenseits der Träume. Seltsame Geschichten vom Anfang unseres Jahrhunderts, Frankfurt a.M. 1990, S. 191. 
Deutschland zu sein". ${ }^{18}$ Die Archäologie der ehemals romantischen Angst hat begonnen.

Neben dieser antiquarisch geprägten novellistischen Phase, die vor allem die stofflichen und narrativen Traditionen epigonal, virtuos oder travestierend aufarbeitet, entstanden allegorische Romane, die ihren phantastischen Plot entweder mit zeitgemäßer Politik oder pseudoreligiöser Lehre auf- und manchmal überladen: Die andere Seite von Alfred Kubin (1877-1959) machte dem bekannten Buchillustrator nicht nur einen Namen als Autor, sondern löste auch eine Mode von utopisch-phantastischen Weltuntergangsromanen aus, unter denen Meyrinks Werke im Kontext des Ersten Weltkriegs herausragen [Der Golem (1915); Das grüne Gesicht (1916); Walpurgisnacht (1917) u.a.]. ${ }^{19}$

Ein denkwürdiges Phänomen ist die explizite Intertextualität und Intermedialität, welche die Gedankenwelt Meyrinks mit Kubins Zeichnungen vernetzt und die Erstlingsromane beider Autoren in eine Art PalimpsestRelation zueinander setzt: Nach Beginn der Niederschrift des Golems um 1907 wurde Kubin beauftragt, Kapitel für Kapitel nach Fertigstellung zu illustrieren. Als Meyrinks Schaffensprozeß stockte, verwendete Kubin 1908 die Zeichnungen zur Illustration seines eigenen Romans, den er kurioserweise unter dem Eindruck einer persönlichen Krise geschrieben hatte, um die stagnierenden Ausdruckmöglichkeiten seiner Kunst durch jene der Literatur zu ergänzen. Der dabei angeblich in einem Guß entstandene Text (EA 1909) diente Meyrink schließlich als Anregung für seine letzten Golem-Kapitel. ${ }^{20}$

Meyrinks Romanwerk vermochte offensichtlich einen zeitgenössischen

${ }^{18}$ Vorwort zu: Geschichten um Mitternacht, Wien-Leipzig 1923.

19 cf. Verna Schuetz: a.a.O., S. 86ff.; Peter Cersowsky: a.a.O., S. 34ff.; Mohammed Qasim: Gustav Meyrink. Eine monographische Untersuchung, Stuttgart 1981; Catherine Mathi: La dramaturgie de Gustav Meyrink. Imaginaire et mystique, Paris 1985; Jan Christoph Meister: Hypostasierung - die Logik mystischen Denkens im Werk G. Meyrinks nach 1907. Eine Studie zur erkenntnistheoretischen Problematik eines phantastischen Oeuvres, Frankfurt a.M. 1986.

${ }^{20}$ Anneliese Hewig: Phantastische Wirklichkeit. Interpretationsstudie zu A. Kubins Roman "Die andere Seite", München 1976, S. 13, Peter Cersowsky: a.a.O., S. 27.

Eine letzte Pointe besteht darin, daß Kubin mit seinem Roman möglicherweise nichts anderes tat, als den ihm mündlich von Meyrink mitgeteilten Urplot des Golem zu realisieren. 
Ich-Kult mit esoterischen Botschaften zwischen Kabbala und Buddha ansprechend zu verbrämen. Die Texte erzählen ekstatische Wege eines Helden zu sich selbst, die sich auch von den Zeitwirren nicht beirren lassen $^{21}$ und nach dem Fatalitätsprinzip einer "verifizierten Legende"22 funktionieren. Dieses willkommene Eskapismus-Angebot machte wahrscheinlich - im Verbund mit einer der aufsehenerregendsten Werbekampagnen der deutschen Verlagsgeschichte - den Golem zum erfolgreichsten deutschsprachigen Roman des Ersten Weltkriegs und Meyrink einmal mehr zum Opfer völkischer Polemik. ${ }^{23}$ Die Katastrophen-Szenarios von K. H. Strobl [Eleagabal Kuperus (1910); Umsturz im Jenseits (1920); Gespenster im Sumpf (1920) u.a.], die von Kapitalisten, Juden, Liberalen und Sozialisten heraufbeschworen werden, sind hingegen deutlich völkisch bis rassistisch geprägt. ${ }^{24}$

Marktleader wie die genannten Autoren gaben Buchreihen und eigene Periodika alter und neuer Phantastik heraus, so z.B. Meyrink die Romane und Bücher der Magie (1921-24) und Strobl die Zeitschrift Der Orchideengarten (1919-21). ${ }^{25}$ Die Blütezeit des Genres bewegte Verlagsunternehmen wie Staackmann (Leipzig), Rikola (Wien), Georg Müller (München) und Drei Masken (Berlin-München-Wien), sich mehr oder weniger auf Phantastik zu spezialisieren. ${ }^{26}$ Und so gilt es auch eine Vielzahl nahezu vergessener phantastischer Autoren von unterschiedlicher Qualität und Gesinnung wiederzuentdecken: Paul Busson (1873-1924), Egmont Colerus (1888-1939), Theodor Däubler (1876-1934), Paul Frank (1885-1976), Paul Leppin (1878-1945), Thaddäus Rittner (1873-1921),

${ }^{21}$ cf. Marianne Wünsch: a.a.O., S. 227 f.

22 Jean Jacques Pollet: Essai sur la littérature fantastique, a.a.O., S. $347 \mathrm{ff}$.

${ }^{23}$ cf. Frans Smit: G. Meyrink. Auf der Suche nach dem Übersinnlichen, München, Wien 1988, S. 113, $126 \mathrm{ff}$.

24 cf. Verna Schuetz: a.a.O., S. 58ff; Günther Wackwitz: Karl Hans Strobl (1877-1946). Sein Leben und sein phantastisch orientiertes Frühwerk, Halle-Wittenberg 1981 [Diss masch.]; Jean Jacques Pollet: "Ecritures fantastiques de l'histoire; K. H. Strobl, A. Lernet-Holenia" in: Austriaca 27 (1988), S. 31-44; Clemens Ruthner: Unheimliche Wiederkehr, a.a.O., S. $64 \mathrm{ff}$.

25 cf. Niteen Gupte: Deutschsprachige Phantastik 1900-1930. Studien und Materialien zu einer literarischen Tendenz unter besonderer Berücksichtigung Österreichs, Essen 1991, S. 30ff.

26 Jens Malte Fischer: a.a.O., S. 118; Niteen Gupte: a.a.O., S. 37ff, 279. 
Otto Soyka (1882-1955), Franz Spunda (1890-1963) u.v.a. ${ }^{27}$ Dabei müßte man auch jene seltsame Affinität der siebziger und achtziger Geburtenjahrgänge zum Phantastischen - meist im Kontext gescheiterter Zivilberufe $^{28}$ oder heruntergekommener Adelsfamilien - einmal genauer literatursoziologisch abklären und auch die regionale Herkunft einbeziehen; häufig handelt es sich nämlich um deutschsprachige Autoren aus den slawisch dominierten Nordterritorien der k.u.k. Monarchie - wird hier Horror zum Medium einer ethnischen Auseinandersetzung?

Andere Autoren, die dem umzirkelten Bezugsfeld nur mit Einschränkungen angehören, wie etwa der Kubin-Freund und groteskhumoristische Österreich-Mythologe Fritz von Herzmanovsky-Orlando (1877-1954), haben es indes bereits zu Gesamtausgaben und - posthumer - Anerkennung gebracht. ${ }^{29}$ Dies gilt auch für einige Autoren, die jene späte dritte Produktionsphase prägen, die über das Abebben der Phantastik-Konjunktur in der zweiten Hälfte der zwanziger Jahre ${ }^{30}$ und in einzelnen Fällen sogar über die Nazi-Diktatur hinausreicht: der PalästinaEmigrant Leo Perutz (1884-1957) und sein Meisterschüler, der spätere PEN-Club-Präsident Alexander Lernet-Holenia (1897-1976), stehen mit ihrem Gesamtwerk für eine kriminalistische, subtil psychologische Phantastik; diese setzt sich mit dem Stellenwert des sich selbst rätselhaften Individuums in einer fatalistisch empfundenen Geschichte auseinander, ohne weitgehend auf Gespenster angewiesen zu sein - dafür aber häufig auf einen konservativ-"habsburgischen Mythos" à la Claudio Magris. ${ }^{31}$

${ }^{27}$ cf. Rein A. Zondergeld: Lexikon der phantastischen Literatur, a.a.O.; Joachim Körber: a.a.O.; Franz Rottensteiner: "German-Language Fantasy since 1900" in: F. N. Magill (Hg.): Survey of Modern Fantasy Literature, Eaglewood Cliffs 1983, Bd. V, S. 2391-2141 und Bd. 4 der DeutschÖsterreichischen Literaturgeschichte von Nagl, Zeidler, Castle, Wien 1937.

${ }^{28}$ Verna Schuetz: a.a.O., S. 99.

${ }^{29} \mathrm{cf}$. Wendelin Schmidt-Dengler: "Groteske und geordnete Wirklichkeit. Anmerkungen zur Prosa F. v. Herzmanovsky-Orlandos" in: Österreich in Geschichte und Literatur 14 (1970), S. 191-201; Monika von Gagern: Ideologie und Phantasmagorie F. v. Herzmanovsky-Orlandos, München 1972 [Diss. masch.]; Susanna Goldberg: "F. v. Herzmanovsky-Orlando. Ein Sammler des Abstrusen und Irrationalen" in: Austriaca 27 (1988), S. 101110.

${ }^{30}$ Jens Malte Fischer: a.a.O., S. $118 f$. 
Ihr belletristisches Traditionsmaterial bekommt die österreichische Phantastik um und nach 1900 vor allem via die französische Literatur zugespielt, die bereits im neunzehnten Jahrhundert in Deutschland offiziell negierte Autoren wie E.T.A. Hoffmann und E.A. Poe begeistert aufgenommen hatte und diese Rezeption nun an das deutschsprachige Publikum weitergibt. ${ }^{32}$

Thre zeitgenössischen Vorläufer findet die gesamte Phantastik-Produktion im deutschsprachigen Raum vor allem im schwülstig-pansexuellen bis satanistischen Werk des Deutschpolen Stanislaw Przybyszewski (18681927), in den grotesken Satiren des später asylierten Süddeutschen Oskar Panizza (1852-1921) und in der Proto-Science Fiction des Preußen Paul Scheerbart (1863-1915), der am Höhepunkt des Naturalismus um 1890 seinen Verlag deutscher Phantasten gründete. ${ }^{33}$ Das Unheimliche dringt aber auch in die kanonisierte österreichische Hochliteratur um 1900 ein, wofür die Spukphänomene in Rilkes Aufzeichnungen des Malte Laurids Brigge (1910) ein gutes Beispiel abgeben, ebenso wie Hofmannsthals

${ }^{31}$ cf. Franziska Müller-Widmer: A. Lernet-Holenia. Grundzüge seines Prosawerks dargestellt am Roman. "Mars im Widder", Wien 1980 [Diss. masch.], Rein A. Zondergeld: "Blaue Augen, nackte Füße oder Die Herrschaft des Anderen. Zu Lernet-Holenia" in: Phaicon 5 (19820, S. 90-100; Dietrich Neuhaus: Erinnerung und Schrecken. Die Einheit von Geschichte, Phantastik und Mathematik im Werk L. Perutz', Frankfurt a.M. 1984; Reinhard Lüth: Drommetenrot und Azurblau. Studien zur Affinität von Erzähltechnik und Phantastik in Romanen von L. Perutz u. A. LernetHolenia, Meitingen 1988; Michael Koseler: "Leo Perutz 'Der Meister des Jüngsten Tages'. Enquête et mise en énigme" in: Austriaca 27 (1988), S. 111-125; Michael Maria Mandelartz: Poetik und Geschichtsphilosophie bei Leo Perutz. Untersuchungen zu seinen Romanen "Die dritte Kugel" und "Nachts unter der steinernen Brücke" nebst einleitenden Bemerkungen zur Ästhetik, zur Theorie der Phantastik und des historischen Romans, Tübingen 1992; Jean Jacques Pollet: "Ecritures fantastique de l'histoire" a.a.O.; Jean Jacques Pollet: Les fatalités ordinaires de Leo Perutz" in: La littérature fantastique, a.a.O., S. 157-169; Jean Jacques Pollet (Hg.): Leo Perutz ou l'ironie de l'Histoire, Rouen: CERA, Publica-tions de l'Université, 1993.

32 cf. Harro Heinz Kühnelt: "E. A. Poe und die phantastische Erzählung im österreichischen Schrifttum von 1900-1920" a.a.O.; Verna Schuetz: a.a.O., S. 24ff.; Jens Malte Fischer: a.a.O., S. 96, 99f.; Peter Cersowsky: a.a.O., S. 101ff.; Jean Jacques Pollet: Essai sur la littérature fantastique du début XXe siècle, a.a.O., S. $31 \mathrm{ff}$.

${ }^{33}$ cf. Verna Schuetz: a.a.O., S. 5; Jean Jacques Pollet: Essai sur la littérature fantastique du début du XXe siècle, a.a.O., S. 39ff. 
Reitergeschichte (1899) und die Novellensammlungen Dämmerseelen (1907) bzw. Masken und Wunder (1912) von Arthur Schnitzler. ${ }^{34}$ Gemeinsam mit den Literaten - und häufig schon vor ihnen - haben sich mehrere Generationen von bildenden Künstlern in Europa inspirieren lassen, sei es durch die Texte einer klassischen Phantastik, sei es von der Kunstgeschichte (Brueghel, Bosch, Callot, Hogarth, Füssli, Goya); unter ihnen etwa die Belgier Félicien Rops (1833-98) und James Ensor (18601949), die Franzosen Gustave Doré (1832-83) und Odillon Redon (18401916), der Deutsche Arnold Böcklin (1827-1901), der Norweger Edvard Munch (1863-1944) und der Engländer Aubrey Beardsley (1872-1898). ${ }^{35}$

Es entstehen Kunstwerke, die scheinbar ein ebenso großer Impuls für die erwähnten österreichischen Phantasten sind wie deren Lektüre: dies ist bei Strobls Böcklin-Bewunderung ersichtlich, wie sie sich anhand seines Vorwortes zu den Geschichten um Mitternacht dokumentieren läßt, vor allem aber beim Poe-Illustrator Kubin, dessen bildnerisches Werk in weiterer Fölge Kafka beinflußt haben dürfte. Nicht außer acht gelassen darf auch die Rolle einer entstehenden zeitgenössischen Kinokultur werden, die sich ausgiebig des Phantastischen bedient, wiewohl ihre einschlägigen Produktionsstätten meist in Deutschland liegen. ${ }^{36}$

Das erwähnte Traditionsmaterial wurde freilich auch abseits des Kulturbetriebs rezipiert. Bemerkenswert ist dabei das bereits erwähnte zeitliche und thematische Nebeneinander von Psychoanalyse und Phantastik, das nachgerade den Vergleich mit einer Parallelaktion herausfordert: ${ }^{37}$ Beide Diskurse loten die Tiefen des menschlichen Bewußtseins aus; es wäre schlüssig zu behaupten, daß der Mangel an einer adäquaten zeitgenössischen Seelenkunde sowohl die Massenpsychologie von Simon Le Bon (1895) und Freud (1921) als auch die phantastischen Romane in der

${ }^{34}$ cf. Gero von Wilpert: Die deutsche Gespenstergeschichte. Motiv-FormEntwicklung, Stuttgart 1994, S. $360 \mathrm{ff}$.

${ }^{35}$ cf. Verna Schuetz: a.a.O., S. 32ff.; Jean Jacques Pollet: Essai sur la littérature fantastique du début du XXe siècle, a.a.O., S. 42ff.

${ }^{36}$ cf. Verna Schuetz: a.a.O., S. 49ff.; Jens Malte Fischer: a.a.O., S. 105f.; Jean Jacques Pollet: Essai sur la littérature fantastique du début du XXe siècle, a.a.O., S. 53ff; u.a.

${ }^{37}$ cf. Jean Jacques Pollet: Essai sur la littérature fantastique du début du XXe siècle, a.a.O., S. 76ff.; Joachim Metzner: "Die Vieldeutigkeit der Wiederkehr. Literaturpsychologische Überlegungen zur Phantastik" in: Phantastik in Literatur und Kunst, a.a.O., S. 80f. 
Nachfolge von Kubins Anderer Seite provozierte ${ }^{38}$ - beispielsweise Otto Soykas Eva Morsini (1923), wo der Revenant von Katharina der Großen die Weltherrschaft an sich reißen möchte. ${ }^{39}$ Die Ergebnisse - nämlich die Beeinflussung von Volksmassen an einer rätselhaft hypnotischen Führerfigur festzumachen - sind überraschend ähnlich (nicht umsonst war Soyka ja auch in Kraus' Fackel Freud-Rezensent gewesen). Umgekehrt gilt freilich, daß das temporäre Interesse der Psychoanalyse für die Phantastik (etwa in den Studien von Otto Rank) von dieser nicht unbedingt erwidert wird, ja sogar - etwa von Kubin selbst - die interpretativen Ansprüche der Freud-Schüler zurückgewiesen werden. ${ }^{40}$

Paradoxerweise könnte man feststellen, daß gerade für eine Literatur, die immer wieder das Ausgeliefertsein des Menschen an Mächte jenseits seiner selbst behauptet, die Existenz eines Un(ter)bewußten im allgemeinen nicht akzeptabel zu sein scheint; ihr Menschenbild bleibt trotz Okkultismus und Anlehnung an die Befindlichkeiten der Epoche einem relativ konventionellen Seelenbegriff verpflichtet. Dies unterscheidet sie auch von internationalen Parallelentwicklungen - wie dem Surrealismus in Frankreich und dem deutschen Expressionismus, mit denen sie Berührungspunkte aufweist.

Bereits in Là bas (1891), der satanistischen Décadence-Bibel des Franzosen Joris Karl Huysmans (1848-1907), ereifern sich die Protagonisten über ihre "bizarre époque": "C'est juste au moment où le positivisme bat son plein, que le mysticisme s'éveille et que les folies de 1'occulte commencent". ${ }^{41}$ Zustandsbestimmungen wie diese gerieren sich zwar als Gegenwartskritik, umzirkeln aber gleichermaßen auch das künstlerische Okkultsubstrat, aus dem die Kultur des frühen zwanzigsten Jahrhunderts genießerisch zehrt.

Ähnliche Worte wie Huysmans findet Stefan Zweig in Die Welt von Gestern $^{42}$ für die frühen zwanziger Jahre im deutschsprachigen Raum. Im symptomatischen Standardwerk von Wilhelm Michel über Das

\footnotetext{
${ }^{38}$ cf. Verna Schuetz: a.a.O., S. $45 \mathrm{ff}$; Marianne Wünsch: a.a.O., S. $121 \mathrm{ff}$.

${ }^{39}$ cf. Clemens Ruthner: Unheimliche Wiederkehr, a.a.O., S. $169 \mathrm{ff}$.

${ }^{40} \mathrm{cf}$. Jean Jacques Pollet: Essai sur la littérature fantastique du début du XXe siècle, a.a.O., S. $76 \mathrm{ff}$.

${ }^{41}$ Paris 1988, S. 290.

${ }^{42}$ Frankfurt a.M. 1994, S. 344ff./ EA 1944.
} 
Teuflische und Groteske in der Kunst heißt es schließlich: "Mischgefühle müssen populär sein in einer Zeit, deren populäres Pathos erst im Werden ist und der zur entschlossenen Verneinung die Lust wie die Kraft mangelt." ${ }^{43}$ Der Untergang einer alten Ordnung mit seinem Strukturwandel zwischen 1900 und 1918 oszilliert solchermaßen ambivalent zwischen Endzeitstimmung und Aufbruch ins Unbekannte, wofür er offensichtlich in der diabolischen Ambiguität der Phantastik ein geeignetes Ausdrucksmedium findet.

Diese ist freilich auch eine Bewegung, die sich eingebettet weiß in die Raffinessen und Extravaganzen des zeitgenössischen Dandytums, wie das exzentrisch bis esoterische Leben des Prager Bankiers und späteren Phantastik-Autors Meyrink zeigt. ${ }^{44}$ Anders verhält es sich mit dem radikal völkischen K. H. Strobl, ${ }^{45}$ der die Verbindungslinie von Phantastik und Heimatkunst darstellt und im Dritten Reich Spitzenfunktionär der Reichsschrifttumskammer wurde. Divergente Biographien wie jene machen deutlich, daß der "Traditionszusammenhang" nach 1900 als Mitkonstituent unseres Genres einmal genauer zu hinterfragen wäre, ${ }^{46}$ ein Kontext, den der Common Sense nach Fischer ${ }^{47}$ mit der Fin de siècle-Kultur gleichsetzt. Ergänzend zu Wünsch ${ }^{48}$ wäre zu beantworten, inwiefern die Phantastik nach 1900 Teil oder Gegenprojekt der literarischen Moderne ist.

Wiewohl es in Österreich keinen eigenständigen Naturalismus wie in Deutschland gegeben hat, teilt die Phantastik mit Bewegungen wie dem Impressionismus, Jugendstil und Expressionismus nicht nur den extravaganten Geschmack, sondern vor allem ihren antinaturalistischen Impetus. ${ }^{49}$ Dieser ist im Falle der phantastischen Literatur durchaus widersprüchlich - übernimmt diese zwar mehr oder minder das realistische Programm einer Mimesis, um es dann jedoch auf positivistisch nicht festmachbare, paranormale Phänomene anzuwenden. ${ }^{50}$ Umgekehrt wirkt

\footnotetext{
${ }^{43}$ München 1911, S. 92. Meyrink, a.a.O.

45 cf. Günther Wackwitz: Karl Hans Strobl, a.a.O.

${ }^{46}$ cf. Peter Cersowsky, a.a.O., S. 22.

${ }^{47}$ Jens Malte Fischer: a.a.O.

${ }^{48}$ Marianne Wünsch: a.a.O.

${ }^{49}$ Niteen Gupte: a.a.O., S. $12 \mathrm{f}$.
}

${ }^{44}$ cf. Mohammed Quasim: Gustav Meyrink, a.a.O.; Frans Smit: Gustav 
die Fremdbestimmung der Figuren durch übernatürliche Mächte in der Phantastik geradezu als eine Mystifizierung der naturalistischen Milieutheorie - die in ihrer eigentlichen Form beispielsweise noch in der Beschreibung des Prager Ghettos in Meyrinks berühmten Golem-Roman (1915) zur Geltung kommt. ${ }^{51}$

Die empiriokritizistische Verabschiedung von Grundaxiomen des Positivismus (Einheit des Subjekts, Faktizität der Außenwelt) durch die Analyse der Empfindungen (1886, $\left.{ }^{2} 1900\right)$ des österreichischen Physikers Ernst Mach (1838-1916) tat das ihrige, um in einer Welt, in der einzig die Sinneseindrücke real sind, auch sogenannte paranormale Wahrnehmungen diskutierbar zu machen. An dieser Stelle ist der zeitgenössische Hang zum Orientalismus und Okkultismus in Betracht zu ziehen: die angesprochene zeitgenössische Konjunktur von asiatisch bis esoterischen Ersatzreligionen, wie etwa Hinduismus und Buddhismus, die Theosophie Helena Blavatskys (1831-1891) und ihresgleichen, die Renaissance magischen Denkens in der Nachfolge der Franzosen Eliphas Levi (18101875) und Alain Kardec, schließlich die Séancen des-Spiritismus,-die_bis in die Zwischenkriegszeit hinein auch durchaus seriöse Intellektuelle wie Thomas Mann in ihren Bann ziehen; ${ }^{52}$ nicht zu vergessen der damalige Trend einer literarischen Umsetzung jüdischer Mystik (z.B. im Golem und in Perutz' Nachts unter der steinernen Brücke), der noch nicht restlos erforscht ist.

Gemeinsam ist der Frühmoderne und der Phantastik auch die antibourgeoise Haltung, die durch ästhetische Skandale (zum Lesen) provozieren will: beiden geht es darum, die Grenzen des empirisch Wahrnehmbaren und sozial Akzeptablen auszureizen, was die Phantastik vor allem in thematischer Hinsicht versucht. Der Hintergrund ist eine zeitgenössische Philosophie des "gesteigerten Lebens",53 das literarische Ergebnis die Kultivierung des Nicht-Alltäglichen, Übersinnlichen, (sexuell) Devianten, Exotischen, Extremen - wie es etwa der deutsche

${ }^{50}$ cf. Niteen Gupte: a.a.O., S. 98f.; Jean Jacques Pollet: Essai sur la littérature fantastique du début du XXe siècle, a.a.O., S. $22 \mathrm{ff.}, 95$.

51 cf. Peter Cersowsky: a.a.O., S. 34ff.

${ }^{52}$ cf. Marianne Wünsch: a.a.O., S. 84ff.; Jean Jacques Pollet: Essai sur la littérature fantastique du début du XXe siècle, a.a.O., S. 61ff., 94f; Peter Cersowsky: a.a.O., S. 47ff; Verna Schuetz: a.a.O., S. 36ff.

${ }^{53}$ Marianne Wünsch: a.a.O., S. 120. 
Phantastik-Autor Hanns Heinz Ewers (1871-1943) in seinem Poe-Essay von 1905 und in den folgenden belletristischen Texten forciert, ähnlich auch Strobl. ${ }^{54}$

Der Parallelismus zwischen Phantastik und etlichen zeitgenössischen Diskursen könnte nahelegen, daß sich die Konjunktur des Imaginären keineswegs nur innerhalb eines zweckfreien Raumes von l'art pour l'art abspielt. Dies würde weitere sozialhistorische und -psychologische Erklärungsmodelle notwendig machen, die jedoch mehr als eine apotropäische Rationalisierungsfunktion dem unangenehm Irrationalen gegenüber haben sollten. Auch stellt sich den Interpreten immer wieder die Frage, wieviel Eskapismus, Politisierung oder Gesellschaftskritik man hinter dem untersuchten Genre vermuten darf. ${ }^{55}$

Für die Epoche 1900-1930 sind unter anderem folgende Motive der Phantastik zu nennen, die auf der Folie von zeitgenössischen Fragestellungen massenhaft Verbreitung finden und auch als Metapher in die Publizistik eindringen: Der apologetische Ich-Kult starker bzw. erstarkender Helden und hypnotischer Demiurgenfiguren, die beliebten apokalyptischen Massenszenarios und fatalistischen Geschichtsbilder wurden bereits erwähnt. Es sind ruhelose, mehr oder minder allegorisierte Auseinandersetzungen mit der gespannten politisch-sozialen Situation, die in Weltkriegen und Umstürzen gipfelt, ${ }^{56}$ und mit der Stellung des zunehmend ohnmächtigen, ja verabschiedeten Individuums in dieser Geschichte, basierend auf einer zeitgenössischen Zivilisationskritik à la Oswald Spengler und Gustave Le Bon. In diesem Zusammenhang wären auch das Motiv der toten Stadt (häufig nach dem Vorbild Prags) und gelegentliche antiamerikanische Züge lesbar, ${ }^{57}$ wo - wie etwa bei Strobl biblischer Untergang und der Irrationalismus einer europäischen Romantik gegen Modernisierung und Industrialisierung aufgewendet werden.

Von Belang ist auch die erotische Überbevölkerung jener Texte v.a. durch

${ }^{54}$ cf. Verna Schuetz: a.a.O., S. 18ff.; Peter Cersowsky: a.a.O., S. 103ff.; Jean Jacques Pollet: Essai sur la littérature fantastique du début de XXe siècle, a.a.O., S. 25ff.; Niteen Gupte: a.a.O., S. 112ff.

${ }^{55}$ cf. Verna Schuetz: a.a.O., S. 57, 68, 95; Jens Malte Fischer: a.a.O.; Winfried Freund: a.a.O.; Clemens Ruthner:Unheimliche Wiederkehr, a.a.O.

${ }^{56}$ cf. Jean Jacques Pollet: Essai sur la littérature fantastique du début du XXe siècle, a.a.O., $110 \mathrm{ff} ., 132 \mathrm{ff}$.

${ }^{57}$ Verna Schuetz: a.a.O., S $193 \mathrm{ff}$. 
fatale Frauen (etwa die Figur der Melitta Lampenbogen in Kubins Anderer Seite, ${ }^{58}$ hinter denen - freilich im Sinne einer Männerliteratur das boomende Interesse für Fragen der Sexualität und die beginnende Frauenbewegung spürbar werden. ${ }^{59}$ Eine Gruppe für sich sind schließlich die Phantome, ${ }^{60}$ künstlichen Menschen und Monstren unterschiedlicher Ausprägung. In ihnen nehmen die Feindbilder jener Zeit Gestalt an, seltener auch die Leitbilder (wie im Golem oder im Grünen Gesicht) in einem Klima des Paradigmenwechsels, das der Zusammenbruch imperial geprägter bürgerlicher Weltordnungen in Mitteleuropa mit sich bringt und das Phantastik einmal mehr zu einem Medium der Unsicherheit und Zerstörung werden läßt.

Ein gutes Beispiel für das Eindringen der Zeitgeschichte in die Phantastik gibt Kubins erwähnter Roman (EA 1909) ab. Diese Anti-Utopie schildert den Aufenthalt seines Ich-Erzählers - Zeichner von Beruf wie Kubin - in einem rätselhaften "Traumreich" in den Tiefen Asiens. Das künstliche Staatengebilde, aus der Nachtseite aller europäischen Kulturen bis zur Mitte des neunzehnten Jahrhunderts zusammengestoppelt, wirkt eigentümlich miefig-antiquiert. Organisiert und zusammengehalten wird dieses soziale Experiment durch den Reichtum und die geheimnisvoll hypnotischen Kräfte einer messianischen Führerfigur - Klaus Patera, der ehemalige Schulkollege des Zeichners. Im apokalyptischen Finale des Romans, an dem ein luziferisch-aufklärerischer amerikanischer Kapitalist namens Hercules Bell maßgeblich beteiligt ist, implodieren indes alle Gegensätze - ein auch in späteren Texten der Phantastik häufiges Bild: "Der Demiurg ist ein Zwitter". ${ }^{61}$ Der Zeichner bleibt als einziger Überlebender in einem Sanatorium zurück.

Neben einer Geographie des Un(ter)bewußten, einer österreichischen Traumideologie in der Nachfolge Grillparzers und Calderons, einer phantastischen Ästhetik mit Anklängen aus unterschiedlichen Philosophien (unter anderem Schopenhauer, Klages und Bahnsen) ist diese dekadente Anti-Utopie auch als sensibler Reflex auf die

${ }^{58}$ cf. Peter Cersowsky: a.a.O., S.83ff., $118 \mathrm{ff}$.

${ }^{59}$ cf. Verna Schuetz: a.a.O., S. 45; Clemens Ruthner: Unheimliche Wiederkehr, a.a.O., S. $126 \mathrm{ff}$.

${ }^{60} \mathrm{cf}$. Clemens Ruthner: Unheimliche Wiederkehr, a.a.O.

${ }^{61}$ Die andere Seite, München 1976, S. 277. 
untergangsgeweihte Stimmung der Monarchie Österreich-Ungarn, jener "Versuchstation des Weltuntergangs" (Karl Kraus), lesbar. ${ }^{62}$ Ähnlich wie das historische "Kakanien" besteht das "Traumreich" aus einer Vielfalt von ethnisch-emotionalen "Heimaten". Dieses Konglomerat wird nur durch Konzepte und eine Vaterfigur zusammengehalten - ein höchst okkultes "Vater-Land", das gewissermaßen die "andere Seite" einer späteren Auseinandersetzung mit dem "habsburgischen Mythos" und der Kaiserfigur in einer realitätskompatiblen österreichischen Literatur darstellt. ${ }^{63}$ Trotz einer gemutmaßten literarischen Vorwegnahme des k.u.k. Zusammenbruchs von 1918 muß Kubins Diskurs aber nicht unbedingt ins Prophetische hochstilisiert zu werden. Es scheint sich vielmehr so zu verhalten, daß sich Phantastik durch ihr programmatisches Hineinragen in die Tiefen des Imaginären, die "Nachtseiten" der menschlichen Existenz, dazu eignet, intuitiv gesellschaftliche Befindlichkeiten schon a priori zu formulieren - im Gegensatz zum Gesellschafts- und Zeitroman, der auf die Distanz der Nachwelt angewiesen ist, wie Musils Mann ohne Eigenschaften (entst. 1925-42) oder Joseph Roths Radetzkymarsch (ersch. 1932).

Phantastik - so könnte man es generell zusammenfassen - reagiert in einer säkularisierten Geschichte sensibel auf ein zeitgenössisches Angebot esoterischen Wissens. ${ }^{64}$ Die Literatur des Imaginären wirkt solchermaßen - zwischen Kolportage und Bewußtseinserweiterung - als eine Art Ersatz-Numinosum für die seit der Aufklärung angekränkelte Religion; gleichzeitig fungiert sie als Krisensymptom ihrer Epoche, als Ausdrucksmedium eines vorhandenen Angst- und Aggressionspotentials, ${ }^{65}$ zur Umsetzung vorhandener Alteritätskonzepte und Tabus, ${ }^{66} \mathrm{zu}$

${ }^{62}$ cf. Verna Schuetz: a.a.O., S. 188ff.; Peter Cersowsky: a.a.O., S. 67ff.; Winfried Freund und Hans Schumacher (Hgg.): Spiegel im dunklen Wort. Analysen zur Prosa des frïhen 20. Jahrhunderts, Frankfurt a.M., Bern 1983; Jean Jacques Pollet: Essai sur la littérature fantastique du début du XXe siècle, a.a.O., S. 301ff; Nicole Berry: "'L'autre côte'. Une lecture psychoanalytique de Kubin" in: Austriaca 27 (1988), S. 127-144.

${ }^{63}$ Die hier präsentierte österreichische Dichotomie Heimat vs. Vaterland stammt von J. G. Helfert aụs der postrevolutionären Ära von 1848ff.

${ }^{64}$ Marianne Wünsch: a.a.O., S. 55.

65 cf. Winfried Freund: "Von der Aggression zur Angst", a.a.O.

${ }^{66}$ cf. Tzvetan Todorov: Introduction à la littérature fantastique, a.a.O., S. 168f; Rein A. Zondergeld: "Zwei Versuche der Befreiung. Phantastische und erotische Literatur" in: Phaicon 2 (1975), S. 64-69. 
einer andauernden Reflexion über die im Zuge einer idealistischbildungsbürgerlichen Ästhetik ausgeschlossenen Bereiche des Häßlichen, Bösen und Jenseitigen. ${ }^{67} \mathrm{Zu}$ dieser kathartisch-kompensatorischen Funktion gesellt sich allerdings noch eine kreative: diese Art von Texten ermöglicht, Diskursschwellen zu überbrücken, Wissenslücken mit den Mitteln der Phantasie zu schließen. Wir sollten sie daher als integralen Wesenszug des Jahrhundertbeginns, der sich nicht pauschal als "reaktionär" oder "trivial" abtun läßt, differenzierter betrachten lernen und gegebenenfalls rehabilitieren.

${ }^{67}$ cf. Hans Richard Brittnacher: Ästhetik des Horrors, a.a.O., S. 7f., S. 11. 